\title{
On the validity of Damköhler's first hypothesis in turbulent Bunsen burner flames: A computational analysis
}

\author{
Nilanjan Chakraborty ${ }^{1}$, Dana Alwazzan ${ }^{1}$, Markus Klein ${ }^{2, *}$, R. Stewart Cant ${ }^{3}$ \\ ${ }^{1}$ School of Engineering \\ University of Newcastle, Claremont Road, Newcastle \\ NE1 7RU, UK
}

Email: nilanjan.chakraboty@ncl.ac.uk ; d.s.a.a.alwazzan@ncl.ac.uk

${ }^{2}$ Universität der Bundeswehr München, Fakultät für Luft- und Raumfahrttechnik, LRT1, Werner-Heisenberg-Weg 39, 85577 Neubiberg, Germany

Email: markus.klein@unibw.de

${ }^{3}$ Cambridge University Engineering Department,

Trumpington Street, Cambridge, CB2 1PZ, United Kingdom

Email: $\underline{\text { rsc10@eng.cam.ac.uk }}$

Colloquium 5: Turbulent Flames (Turbulent-Premixed)

Running title: Damköhler's first hypothesis in turbulent premixed Bunsen flames

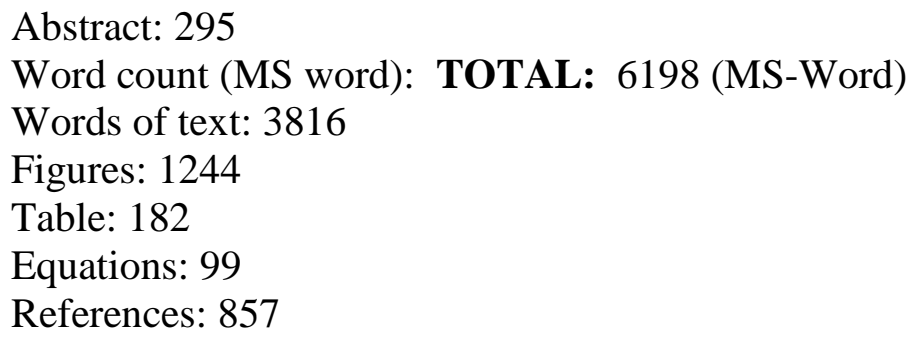

No figure need to be printed in colour in the hard copy. All figures need to be printed in colour in the online version. 


\section{ABSTRACT}

The validity of Damköhler's first hypothesis, which relates the turbulent flame speed to turbulent flame surface area under the condition where the integral length scale of turbulence is greater than the flame thickness, has been assessed using three-dimensional Direct Numerical Simulations (DNS) of turbulent premixed Bunsen burner flames over a range of values of Reynolds number, pressure and turbulence intensity. It has been found for the Bunsen configuration that the proportionality between volumeintegrated burning rate and the overall flame surface area is not strictly maintained according to Damköhler's first hypothesis. The discrepancy is found to originate physically from the local stretch rate dependence of displacement speed, and this helps to explain differences observed previously between flames with and without mean curvature. Approximating the local flame propagation speed with the unstrained laminar flame speed is shown to be inaccurate, and can have a significant influence on the prediction of the overall burning rate for flames with non-zero mean curvature. Using a twodimensional projection of the actual scalar gradient for flame area evaluation is shown to exacerbate the loss of proportionality between volume-integrated burning rate and the overall flame surface area. The current analysis identifies the conditions under which Damköhler's hypothesis remains valid and the necessary correction for non-zero mean flame curvature. Further, it has been demonstrated that surface-weighted stretch effects on displacement speed need to be accounted for in order to ensure the validity of Damköhler's hypothesis under all circumstances. Finally, it has been found that the volumeintegrated density-weighted scalar dissipation rate remains proportional to the overall burning rate for all flames considered here irrespective of the value of Reynolds number, pressure and turbulence intensity. However, this proportionality is lost when the scalar dissipation rate is evaluated using the two-dimensional projection of the actual scalar gradient.

Keywords: Damköhler's first hypothesis, burning rate, flame surface area, Bunsen burner premixed flame, Direct Numerical Simulations 


\section{INTRODUCTION}

In premixed turbulent combustion modelling, the turbulent flame speed $S_{T}$ is a quantity of fundamental importance. The turbulent flame speed is defined as $S_{T}=\int_{V} \dot{W} d V /\left(\rho_{0} A_{L}\right)$ where $\dot{w}$ is the production rate of the reaction progress variable $c, \rho_{0}$ is the unburned gas density and $A_{L}$ is the projected area in the direction of flame propagation. Several authors [1-10] have analysed the statistical behaviour of the turbulent flame speed and proposed models for it. The modelling of $S_{T}$ often invokes Damköhler's first hypothesis [11], which relates the turbulent flame speed to the turbulent flame surface area $A_{T}$ according to:

$$
S_{T} / S_{L}=A_{T} / A_{L}
$$

Here $S_{L}$ denotes the unstrained laminar burning velocity. Although eq. 1 was not referred to as first hypothesis in the original paper by Damköhler [11], this terminology has become commonly used in the literature [12-14]. In the flamelet regime of turbulent premixed combustion, it is often assumed that the burning rate per unit area can be approximated by the corresponding value for unstrained laminar premixed flames, which is given by $\rho_{0} S_{L}$. Thus, the turbulent burning rate can be approximated by $\rho_{0} S_{L} A_{T}$, which upon division by the mean projected area in the direction of flame propagation yields $\rho_{0} S_{T}$ and forms the basis of eq. 1 . Thus the modelling of turbulent flame speed (and hence the overall burning rate) translates to the modelling of flame surface area. This is utilised in the Flame Surface Density (FSD) model [15] through the expression:

$$
\overline{\dot{w}+\nabla \cdot\left(\rho D_{c} \nabla c\right)}=\overline{\left(\rho S_{d}\right)_{s}} \Sigma_{g e n}
$$

where $D_{c}$ is the diffusivity of the reaction progress variable, $S_{d}=|\nabla c|^{-1}(D c / D t)$ is the displacement speed, $\quad \Sigma_{g e n}=\overline{|\nabla c|}$ is the generalised FSD and $\overline{(Q)_{s}}=\overline{Q|\nabla c|} / \overline{|\nabla c|}$ is the surface-weighted value of a general variable $Q$ with the overbar denoting a Reynolds averaging 
or LES filtering operation as appropriate [15]. As the total turbulent flame area is invariant with respect to Reynolds averaging/LES filtering, $A_{T}$ can be expressed as: $A_{T}=\int_{V}|\nabla c| d V=$ $\int_{V} \overline{|\nabla c|} d V=\int_{V} \Sigma_{g e n} d V$. Often in experimental analyses [8,9,16-21] the flame area is determined by edge detection algorithms based on 2D measurements. However, Chen and Bilger [20] demonstrated that the associated errors can be considerable, which motivated them to measure 3D scalar gradients. In order to avoid any ambiguity, in the present analysis the flame area is evaluated by volume-integrating $|\nabla c|$ or $\Sigma_{g e n}$.

Volume-integrating the left hand side of eq. 2 and using the definition of the turbulent flame speed yields $\rho_{0} S_{T} A_{L}=\int_{V} \dot{w} d V=\int_{V} \dot{\dot{w}} d V=\int_{V} \overline{\left(\rho S_{d}\right)_{s}} \Sigma_{\text {gen }} d V, \quad$ since $\int_{V} \overline{\nabla \cdot\left(\rho D_{c} \nabla c\right)} d V$ vanishes due to the divergence theorem. This gives rise to:

$$
\rho_{0} S_{T} A_{L}=\int_{V} \dot{w} d V=\frac{\int_{V} \overline{\left(\rho S_{d}\right)_{s}} \Sigma_{g e n} d V}{\int_{V} \Sigma_{g e n} d V} \int_{V} \Sigma_{g e n} d V=\frac{\int_{V} \overline{\left(\rho S_{d}\right)_{s}} \Sigma_{g e n} d V}{\int_{V} \Sigma_{g e n} d V} A_{T}
$$

It is worth noting that ${\overline{\left(\rho S_{d}\right)_{S}}}_{S}$ is often approximated as ${\overline{\left(\rho S_{d}\right)_{S}}}_{S}=\rho_{0} S_{L}$, which reduces eq. 3 to a statement of Damköhler's first hypothesis, i.e. eq. 1. Eqs. 1 and 3 suggest that for a given burner $\int_{V} \dot{w} d V$ is expected to be proportional to $\int_{V}|\nabla c| d V$ and their ratio is expected to be constant and equal to $\rho_{0} S_{L}$ for a given fuel-air mixture and unburned gas condition for $\overline{\left(\rho S_{d}\right)_{s}}=\rho_{0} S_{L}$. However, it is worth noting that eq. 1 is strictly valid only when $\overline{\left(\rho S_{d}\right)_{s}}=$ $\rho_{0} S_{L}$. In the context of Reynolds Averaged Navier Stokes (RANS) simulations $\overline{\dot{w}}$ is often expressed in terms of FSD as $\overline{\dot{w}}=I_{0} \rho_{0} S_{L} \Sigma_{\text {gen }}[3,22]$ where $I_{0}$ is the stretch factor. It is important to note that $I_{0}$ is not a constant and depends on local mean values of strain rate and curvature [3,22]. This leads to $\rho_{0} S_{T} A_{L}=\int_{V} \overline{\dot{w}} d V=\int_{0} I_{0} \rho_{0} S_{L} \Sigma_{g e n} d V \neq$ $\rho_{0} I_{0} S_{L} \int_{0} \Sigma_{g e n} d V\left(=\rho_{0} I_{0} S_{L} A_{T}\right)$. Hence eq. 1 is not satisfied even if the unstrained flame speed is replaced with $I_{0} S_{L}$. Indeed, experimental data by Gülder [16] has demonstrated that 
the ratio of $\int_{V} \dot{w} d V$ and $\int_{V} \rho_{0} S_{L}|\nabla c| d V$ is not equal to unity in turbulent premixed Bunsen burner flames.

In the flamelet regime, the mean reaction rate $\overline{\dot{w}}$ can be modelled instead using scalar dissipation rate $\widetilde{N}_{c}=\overline{\rho D \nabla c . \nabla c} / \bar{\rho}$ as $[23,24]$ :

$$
\overline{\dot{w}}=2 \bar{\rho} \widetilde{N}_{c} /\left(2 c_{m}-1\right)
$$

where $c_{m}=\int_{0}^{1}[\dot{w} c]_{L} f(c) d c /[\dot{w}]_{L} f(c) d c$, the subscript ' $L$ ' indicates laminar flame values and $f(c)$ is the burning mode probability density function (pdf). Bray [23] demonstrated that $c_{m}$ does not depend significantly on the choice of $f(c)$ as long as a continuous function is presumed for $f(c)$. The quantity $c_{m}$ is constant for a homogeneous fuel-air mixture composition. Equation 4 implies that $\int_{V} \dot{w} d V$ is proportional to $\int_{V} \bar{\rho} \widetilde{N}_{c} d V=\int_{V} \rho N_{c} d V$ and their ratio is expected to be constant and equal to $2 /\left(2 c_{m}-1\right)$ for a given mixture composition. The main objectives of this paper are:

- To discuss whether eq. 1 remains identically valid for a general situation and to determine its range of validity based on the simulations presented in this work

- To discuss challenges which are encountered during experimental assessment of eq. 1

In the present paper, the validity of eqs. 1, 3 and 4 is assessed based on Direct Numerical Simulation (DNS) data for turbulent premixed Bunsen flames within the flamelet regime of combustion for different values of pressure, Damköhler, Karlovitz and turbulent Reynolds numbers. The Bunsen flame configuration can be realised in laboratory-scale experiments $[8$, 9,16-21] and is computationally affordable, despite being more computationally demanding than conventional decaying turbulence DNS in a box. 


\section{NUMERICAL IMPLEMENTATION}

For the purpose of this analysis, nine turbulent premixed Bunsen burner flames have been considered and these flames are taken from a database consisting of 15 different cases [25]. The chemical mechanism is simplified here using a single step irreversible reaction for the sake of computational economy in the interests of a detailed parametric analysis. It has been demonstrated in the past that flame propagation statistics obtained from detailed chemistry simulations [26,27] can be well captured using simple chemistry [28,29]. Furthermore, models which have been proposed based on the analysis of simple chemistry DNS of turbulent premixed flames with simplified transport have the potential to be valid even in the presence of detailed chemistry and transport (subject to minor adjustments) [30,31]. In summary, the assumption of a single step global reaction rate does not affect the qualitative nature of the results presented in this work because the essential physics affecting the local strain and curvature dependence of displacement speed can be captured using simple chemistry [28, 29].

The simulation parameters are listed in Table 1 . These include the Reynolds number $R e=$ $U_{B} D / v_{u}$ based on the bulk inlet velocity $U_{B}$, nozzle diameter $D$, and the unburned-gas kinematic viscosity $v_{u}$; the turbulent Reynolds number $R e_{t}=u^{\prime} l / v_{u}$; the normalised turbulent root-mean-square (rms) velocity fluctuation $u^{\prime} / S_{L}$; normalized mean inlet velocity $U_{B} / S_{L}$; integral length scale to thermal flame thickness ratio $l / \delta_{t h}$; integral length scale to Bunsen burner nozzle diameter ratio $l / D$; Damköhler number $D a=l S_{L} / \delta_{t h} u^{\prime}$; and Karlovitz number $K a=\left(u^{\prime} / S_{L}\right)^{3 / 2}\left(l / \delta_{t h}\right)^{-1 / 2}$. Here $\delta_{t h}=\left(T_{a d}-T_{0}\right) / \max |\nabla T|_{L}$ is the thermal flame thickness with the adiabatic flame temperature $T_{a d}$ and the reactant temperature $T_{0}$. Note that the subscript ' $L$ ' refers to the unstrained laminar flame quantities. The heat release parameter $\tau=\left(T_{a d}-T_{0}\right) / T_{0}$ and the Zel'dovich number $\beta=T_{a c}\left(T_{a d}-T_{0}\right) / T_{a d}^{2}$ are taken to be 4.5 and 6.0 respectively, and $T_{a c}$ is the activation temperature. Standard values of Prandtl number 
$(\operatorname{Pr}=0.7)$ and ratio of specific heats $\left(\gamma_{g}=1.4\right)$ have been used. All non-dimensional numbers are quoted as the inlet values.

The high pressure Bunsen flames have been simulated by adjusting the viscosity and the Arrhenius parameters in such a way that $S_{L} \sim P^{-0.5}$, and $v_{u} \sim P^{-1}$ are satisfied, similar to the behaviour of methane-air flames [32]. As a result of this, the Zeldovich flame thickness $\delta_{Z}$ scales as $\delta_{Z} \sim P^{-0.5}$ (where $\delta_{Z}=\alpha_{T 0} / S_{L}$ with $\alpha_{T 0}$ being the thermal diffusivity in the unburned gas) and the numerical resolution must be adjusted accordingly. The dimensions of the simulation domain are kept unchanged for all cases considered here. The cubic domain is of size $50 \delta_{t h} \times 50 \delta_{t h} \times 50 \delta_{t h}$ for the $P=P_{0}$ flame, $112 \delta_{t h} \times 112 \delta_{t h} \times 112 \delta_{t h}$ for $P=$ $5 P_{0}$ and $159 \delta_{t h} \times 159 \delta_{t h} \times 159 \delta_{t h}$ for $P=10 P_{0}$. The corresponding uniform Cartesian meshes contain $250 \times 250 \times 250,560 \times 560 \times 560$ and $795 \times 795 \times 795$ points. The burner nozzle diameter corresponds roughly to half the domain length. It can be seen from Table 1 that cases A-C have different values of $R e_{D}$ and $R e_{t}$ in spite of having same set of values of $u^{\prime} / S_{L}, U_{B} / S_{L}$, and $l / D$ because of the change in kinematic viscosity with pressure. The low pressure cases $\mathrm{D}$ and $\mathrm{E}$ have the same value of $R e_{t}$ as that of the high pressure case $\mathrm{C}$ and in order to match the turbulent Reynolds number the value of $u^{\prime} / S_{L}$ is modified for case $\mathrm{D}$, whereas $l / D$ is modified in case E. Cases $\mathrm{C}$ and $\mathrm{E}$ have same values of $u^{\prime} / S_{L}$ and $l / \delta_{t h}$, and thus they occupy the same location on the regime diagram. The high pressure cases E-I are chosen in such a manner that the effects of $u^{\prime} / S_{L}$ and $l / D$ variations can be analysed. The relative positions of these cases on the combustion regime diagram by Peters [33] are shown in Fig. 1. Cases A, B, C, E, H and I fall on the boundary of the wrinkled flamelets and corrugated flamelets regimes, whereas case F represents the corrugated flamelets regime and cases $D$ and $G$ represent the thin reaction zones regime. 


\section{Table 1}

The turbulence inlet flow parameters for the considered cases

\begin{tabular}{|c|c|c|c|c|c|c|c|c|c|}
\hline Case & $\boldsymbol{P} / \boldsymbol{P}_{\mathbf{0}}$ & $\boldsymbol{R e}_{\boldsymbol{D}}$ & $\boldsymbol{R e}_{\boldsymbol{t}}$ & $\boldsymbol{U}_{\boldsymbol{B}} / \boldsymbol{S}_{\boldsymbol{L}}$ & $\boldsymbol{u}_{\text {inlet }}^{\prime} / \boldsymbol{S}_{\boldsymbol{L}}$ & $\boldsymbol{l} / \boldsymbol{D}$ & $\boldsymbol{l} / \boldsymbol{\delta}_{\boldsymbol{t h}}$ & $\boldsymbol{K a}$ & $\boldsymbol{D a}$ \\
\hline $\mathrm{A}$ & 1.0 & 399 & 13.30 & 6.0 & 1.0 & $1 / 5$ & 5.20 & 0.45 & 5.00 \\
\hline $\mathrm{B}$ & 5.0 & 892 & 29.26 & 6.0 & 1.0 & $1 / 5$ & 11.40 & 0.30 & 11.40 \\
\hline $\mathrm{C}$ & 10.0 & 1262 & 41.22 & 6.0 & 1.0 & $1 / 5$ & 16.13 & 0.25 & 16.13 \\
\hline D & 1.0 & 399 & 41.22 & 6.0 & 3.1 & $1 / 5$ & 5.20 & 2.40 & 1.670 \\
\hline E & 1.0 & 399 & 41.22 & 6.0 & 1.0 & $3 / 5$ & 16.13 & 0.25 & 16.13 \\
\hline F & 10.0 & 1262 & 82.45 & 6.0 & 2.0 & $1 / 5$ & 16.13 & 0.7 & 8.00 \\
\hline G & 10.0 & 1262 & 164.9 & 6.0 & 4.0 & $1 / 5$ & 16.13 & 2.00 & 4.00 \\
\hline H & 10.0 & 1262 & 10.31 & 6.0 & 1.0 & $1 / 20$ & 3.87 & 0.51 & 3.87 \\
\hline I & 10.0 & 1262 & 164.9 & 6.0 & 1.0 & $4 / 5$ & 61.90 & 0.13 & 61.90 \\
\hline
\end{tabular}

The simulations have been conducted using the compressible 3D DNS code SENGA [34] in which the governing equations are solved in non-dimensional form. The spatial differentiation for internal grid points is carried out using a $10^{\text {th }}$ order central differencing scheme which gradually reduces to a one-sided $2^{\text {nd }}$ order scheme at non-periodic boundaries. Timeadvancement is carried out using a $3^{\text {rd }}$ order explicit low storage Runge-Kutta scheme. Inflow data has been generated using a modified version of the methodology proposed by Klein et al. [35] where the Gaussian filter in the axial direction has been replaced by an autoregressive AR1 process in order to avoid excessive filter length in this direction caused by the small time step in the compressible flow solver. The reacting flow field is initialised using an unstrained premixed laminar flame solution which is specified as a function of radius from the nozzle centre resulting in a hemispherical scalar field located at the inflow. The mean velocity profile after the nozzle exit has been approximated by a hyperbolic-tangent like distribution. The other boundaries are taken to be partially non-reflecting and are specified using the Navier-Stokes Characteristic Boundary Conditions (NSCBC) formulation [36]. The simulation time, when statistics are first considered, is chosen to be larger than the maximum of two flow-through and two eddy-turnover times. 


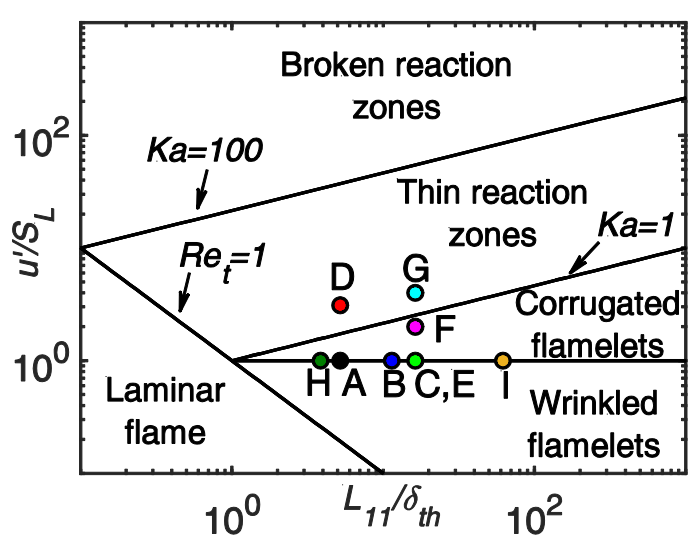

Fig. 1: The inlet conditions of Bunsen burner flames on the combustion regime diagram.

\section{RESULTS \& DISCUSSION}

\section{Description of turbulent reacting flow field}

Instantaneous views of reaction progress variable $c$ isosurfaces for cases $\mathrm{A}, \mathrm{C}$ and $\mathrm{E}$, and the corresponding distributions of normalised velocity magnitude $|\vec{u}| / S_{L}$ at the central mid-plane are shown in Fig. 2. It can be seen that the flame becomes increasingly wrinkled from case A to case $\mathrm{C}$ (case B is not shown for brevity) with an increase in both Reynolds number and pressure. Moreover, the nature of flame wrinkling in case $\mathrm{C}$ is different from case $\mathrm{E}$ in spite of both cases $\mathrm{C}$ and $\mathrm{E}$ sharing the same location on the regime diagram. It has been discussed elsewhere [25] that an increase in pressure reduces the normalised cut-off length for DarrieusLandau (DL) instability given by $\lambda_{c} / D\left(\lambda_{c} / \delta_{Z}\right)$. Thus the effects of DL instability are likely to be prominent for flames at elevated pressures (e.g. case C) but these effects are absent in flames at low pressures (e.g. cases A and E). The distribution of $|\vec{u}| / S_{L}$ shows high values at the flame tip. This is due to the focussing of heat at the flame tip giving rise to significant flow acceleration, and a qualitatively similar behaviour has been observed in other cases. 

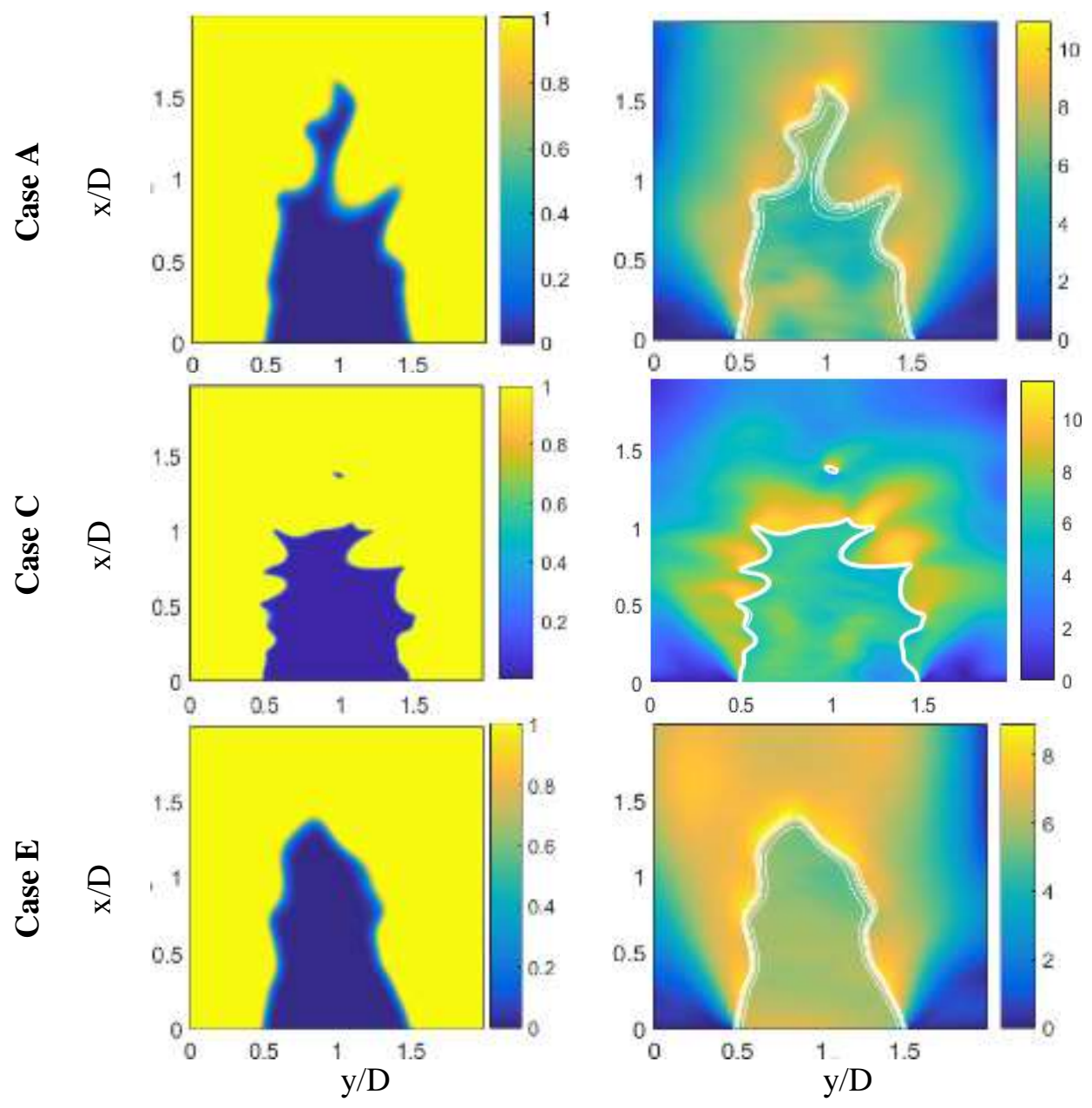

Fig. 2: Instantaneous view of (a) reaction progress variable $c$ ( $1^{\text {st }}$ column) and (b) normalised velocity magnitude $|\vec{u}| / S_{L}\left(^{\text {nd }}\right.$ column) in the central mid-plane for cases $\mathrm{A}$, C and E.

\section{Overall burning rate and flame surface area and their inter-relations}

Figure 3 shows the mean values of normalised burning rate $\Omega=\int_{V} \dot{w} d V / \rho_{0} S_{L} D^{2}$ (open circles) and normalised flame surface area $S=\int_{V}|\nabla c| d V / D^{2} \quad$ (star symbols) based on averaging different realisations in time for all cases considered here. Here, $\Omega$ is evaluated using $\int_{V} \dot{w} d V / \rho_{0} S_{L} D^{2}$ in order to avoid any uncertainty in the evaluation of projected flame surface area. According to eq. 1 , the quantities $\Omega$ and $S$ are expected to be identical to each other but Fig. 3 shows that these quantities remain close but not identical to each other. This can further be verified from Fig. 4 where the values of $\langle\Omega\rangle /\langle S\rangle$ are shown (dark blue columns) where $\langle\ldots\rangle$ 
indicates the average over different realisations. The value of $\langle\Omega\rangle /\langle S\rangle$ is greater than unity for all cases, and the deviation from unity is almost $10 \%$ for some cases. The disagreement between $\langle\Omega\rangle$ and $\langle S\rangle$ is consistent with previous experimental observations by Gülder [16], in which the magnitude of the discrepancy is somewhat larger.

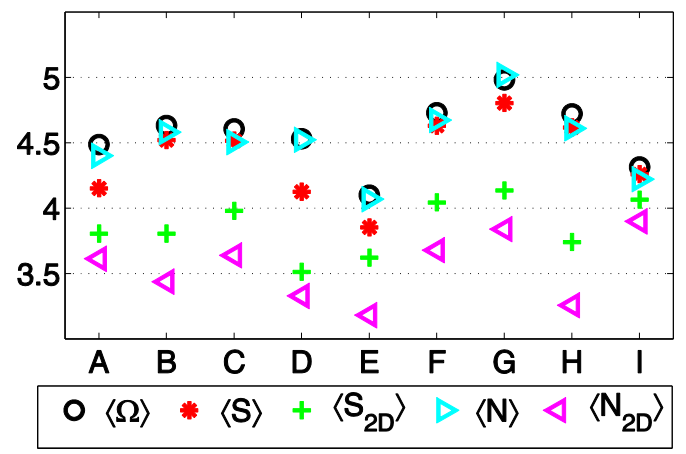

Fig. 3: Variations of $\langle\Omega\rangle,\langle S\rangle,\left\langle S_{2 D}\right\rangle,\langle N\rangle,\left\langle N_{2 D}\right\rangle$ for cases A-I based on averaging different realisations.

For the sake of completeness, it is worth noting that the results presented in this paper do not change if the normalised flame surface area $S$ is evaluated based on the fine-grained FSD $\Sigma=$ $\overline{|\nabla c| \delta\left(c-c^{*}\right)}$ [37] using the expression $S=\int_{V} \Sigma d V / D^{2}$. This has been checked for the isosurfaces $c^{*}=0.5$ and 0.8 (not shown here). Moreover, the maximum difference between the flame areas evaluated using $\int_{V} \Sigma_{\text {gen }} d V$ and by careful, DNS data based, edge detection of $c^{*}=0.5$ and 0.8 isosurfaces has been found to be within $2-3 \%$ for the cases considered here.

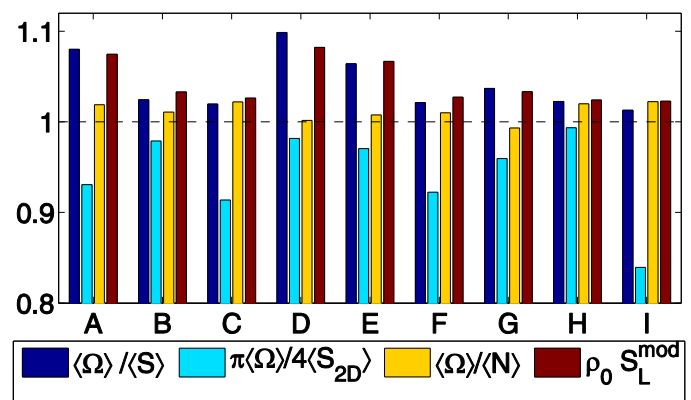

Fig. 4: Variations of $\langle\Omega\rangle /\langle S\rangle, \pi\langle\Omega\rangle / 4\left\langle S_{2 D}\right\rangle,\langle\Omega\rangle /\langle N\rangle$ and $\rho_{0} S_{L}^{\text {mod }}$ for cases A-I. 
It is often difficult to carry out three-dimensional measurements of the scalar gradient magnitude, and thus it is worthwhile to consider the implications of two-dimensional measurements (e.g. Refs. [1,3,8,9,16-19,21] and references therein). The magnitude of the twodimensional projection of the actual gradient of reaction progress variable is evaluated here using the instantaneous radial and axial gradients of $c$. The mean values of $S_{2 D}=$ $\int_{V}|\nabla c|_{2 D} d V / D^{2}$ for all cases are shown in Fig. 3 (cross symbols), where the subscript '2D' denotes quantities evaluated using $2 \mathrm{D}$ gradients. However, the absence of the gradient in one direction reduces the magnitude of $S_{2 D}$ in comparison to $S$. Thus, $\langle\Omega\rangle /\left\langle S_{2 D}\right\rangle$ takes a value which is significantly greater than unity (not shown). It has been shown elsewhere in previous semianalytical and DNS studies [38,39] that the ratio of actual generalised FSD to its 2D counterpart is given by $4 / \pi$ and it can be seen from Fig. 4 that $\pi\langle\Omega\rangle / 4\left\langle S_{2 D}\right\rangle$ (pale blue columns) is indeed close to $\langle\Omega\rangle /\langle S\rangle$ (dark blue columns) although a notable underprediction is visible especially for cases $\mathrm{C}, \mathrm{F}$ and I. It is worth noting that the correction factor $4 / \pi$ was derived based on the assumption of isotropy of the angle between the local flame normal and the normal vector of the measurement plane $[34,35]$, but this may not be valid in the flames where the effects of DL instability are strong [25]. Thus, $\pi\langle\Omega\rangle / 4\left\langle S_{2 D}\right\rangle$ underpredicts $\langle\Omega\rangle /\langle S\rangle$ in cases $\mathrm{C}, \mathrm{F}$ and I but this is not prominent for cases $\mathrm{G}$ and $\mathrm{H}$ because of high turbulence intensity and small length scale, respectively. The experimental analysis by Zhang et al. [21] indicated a considerable variation of the correction factor between $2 \mathrm{D}$ and $3 \mathrm{D}$ measurements of FSD, under the assumption that Damköhler's first hypothesis is valid. This assumption may not be strictly true and moreover, the uncertainty associated with experimental determination of flame area based on 2D edge detection [20] may act to increase the observed variation still further. 
Finally, it is shown in Fig. 3 that the quantity $N=2 \int_{V} \rho N_{c} d V /\left[\left(2 c_{m}-1\right) \rho_{0} S_{L} D^{2}\right]=$ $2 \int_{V} \rho D_{c}|\nabla c|^{2} d V /\left[\left(2 c_{m}-1\right) \rho_{0} S_{L} D^{2}\right]$ (right-pointing triangles) satisfactorily captures the behaviour of $\Omega=\int_{V} \dot{w} d V / \rho_{0} S_{L} D^{2}$ (open circles) irrespective of the values of $P, u^{\prime} / S_{L}$ and $l / \delta_{t h}$. The ratio $\langle\Omega\rangle /\langle N\rangle$ is shown in Fig 4 and is seen to be close to unity for all cases. By contrast, $\quad N_{2 D}=2 \int_{V} \rho D_{c}|\nabla c|_{2 D}^{2} d V /\left[\left(2 c_{m}-1\right) \rho_{0} S_{L} D^{2}\right]$ (Fig 3, left-pointing triangles) significantly underpredicts $\Omega$ (open circles). The results for reaction rate closure based on scalar dissipation rate (SDR) indicate that according to $\overline{\dot{w}}=\overline{\left(\rho S_{d}\right)_{s}} \Sigma_{g e n}=2 \bar{\rho} \widetilde{N_{c}} /\left(2 c_{\mathrm{m}}-1\right)$ the volume-integrated $\overline{\dot{w}}$ remains directly proportional to the volume-integrated value of $\bar{\rho} \widetilde{N_{c}}$, whereas volume-integrated $\overline{\dot{W}}$ does not remain strictly proportional to volume-integrated $\Sigma_{g e n}$ because $\overline{\left(\rho S_{d}\right)_{s}}$ does not necessarily remain constant. Indeed the local and area-weighted stretch rate dependence of displacement speed needs to be accounted for in order to interlink the volume-integrals of $\Sigma_{g e n}$ and $\overline{\dot{W}}$. This will be explained in the next section.

\section{Physical explanations for the observed behaviour}

The displacement speed in turbulent premixed flames is affected by local strain rate and curvature [26-29] and thus ${\overline{\left(\rho S_{d}\right)_{s}}}_{s}$ should not be treated as a constant quantity. This can be substantiated from Fig. 5 where the variations of the mean values of $\rho S_{d}|\nabla c|$ and $\rho_{0} S_{L}|\nabla c|$ conditional upon $c$ are shown for cases $\mathrm{A}$ and $\mathrm{C}$ together with the corresponding standard deviations. Figure 5 shows that the mean values of $\rho S_{d}|\nabla c|$ and $\rho_{0} S_{L}|\nabla c|$ are not equal to each other and the extent of agreement between $S_{d}|\nabla c|$ and $\rho_{0} S_{L}|\nabla c|$ changes within the flame brush. Furthermore, Fig. 5 shows that the standard deviations of $\rho S_{d}|\nabla c|$ and $\rho_{0} S_{L}|\nabla c|$ are significantly different and hence the local behaviours of these quantities are expected to be considerably different. Fig. 5 is based on the samples taken from the whole flame but the results look qualitatively similar if different axial locations are considered. 


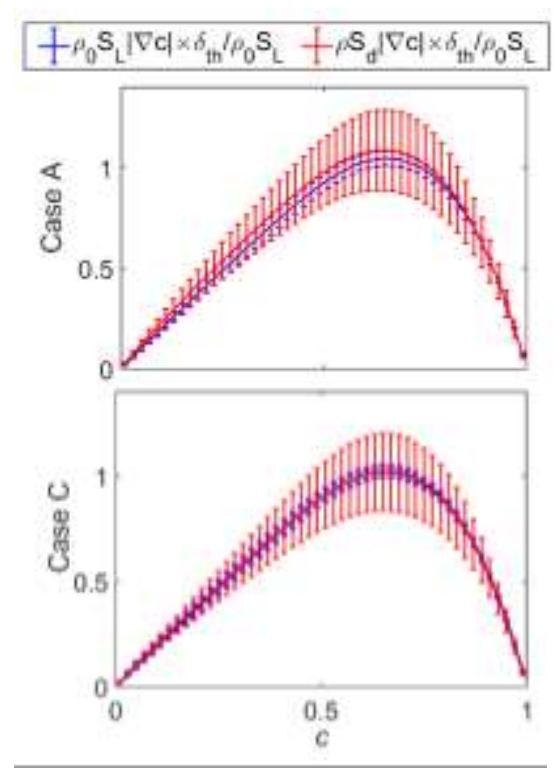

Fig. 5: $\rho S_{d}|\nabla c| \times \delta_{t h} / \rho_{0} S_{L}$ and $\rho_{0} S_{L}|\nabla c| \times \delta_{t h} / \rho_{0} S_{L}$ conditional upon $c$ for cases A and $\mathrm{C}$. The bars indicate the standard deviation of the respective quantities.

Hence the volume integral $\int_{V} \overline{\left(\rho S_{d}\right)_{S}} \Sigma_{g e n} d V$ leads to $\rho_{0} S_{L}^{\prime} \int_{V} \Sigma_{g e n} d V=\rho_{0} S_{L}^{\prime} A_{T}$ where $S_{L}^{\prime}$ is a modified flame speed given by:

$$
S_{L}^{\prime}=\int_{V}{\overline{\left(\rho S_{d}\right)}}_{s} \Sigma_{g e n} d V / \int_{V} \rho_{0} \Sigma_{\text {gen }} d V
$$

This expression implicitly accounts for the volume-integrated FSD-weighted stretch rate dependence of the local displacement speed. Moreover, in the context of the reaction rate closure model $\overline{\dot{W}}=I_{0} \rho_{0} S_{L} \Sigma_{g e n}$ the modified flame speed $S_{L}^{\prime}$ is given by

$$
S_{L}^{\prime}=S_{L} \int_{V} I_{0} \Sigma_{\text {gen }} d V / \int_{V} \Sigma_{\text {gen }} d V
$$

which suggests that $S_{L}^{\prime}$ becomes identical to $S_{L}$ when $\int_{V} I_{0} \Sigma_{\text {gen }} d V \approx \int_{V} \Sigma_{\text {gen }} d V$.

It is worth noting a flame speed similar to $S_{L}^{\prime}$ was used by Chakraborty and Cant [40] for LES modelling of FSD. Furthermore, this is consistent with recent findings by Sabelnikov et al. [41] which suggest that the assumption $\overline{\left(\rho S_{d}\right)_{s}}=\rho_{0} S_{L}$ yields incorrect behaviour of FSD. Thus, it is more appropriate to express turbulent flame speed as $S_{T}=S_{L}^{\prime} A_{T} / A_{L}$ instead of using eq. 1 . 
However, it has been shown in several previous DNS studies involving statistically planar flames that $\int_{V} \dot{w} d V$ remains almost equal to $\rho_{0} S_{L} \int_{V}|\nabla c| d V$ even for large values of $u^{\prime} / S_{L}$ [42-44]. It is worth noting that statistically planar flames also experience stretch under turbulent conditions, but the closeness of $\int_{V} \dot{w} d V$ and $\rho_{0} S_{L} \int_{V}|\nabla c| d V$ in these cases suggests that the stretch rate dependence of $I_{0}$ mostly disappears upon evaluating the integral $\int_{V} I_{0} \Sigma_{\text {gen }} d V$, and hence $\int_{V} I_{0} \Sigma_{\text {gen }} d V \approx \int_{V} \Sigma_{\text {gen }} d V$ in planar flames. However, the inequality between $\Omega$ and $S$ in turbulent Bunsen burner flames suggests that the assumption $\int_{V} I_{0} \Sigma_{\text {gen }} d V \approx \int_{V} \Sigma_{\text {gen }} d V$ is rendered invalid when the flame has a mean curvature. In fact the experimental data of Zhang et al. [21] indicated that $I_{0}$ may show considerable variation even though its mean value remains of the order of unity. The dependence of $S_{d}$ on flame curvature $\kappa_{m}$ can be modelled according to $\rho S_{d}=\rho_{0}\left(S_{L}-D_{M} \kappa_{m}\right)$ where $D_{M}$ is the Markstein diffusivity [45] and $\kappa_{m}=1 / 2 \nabla \cdot(-\nabla c /|\nabla c|)$, defined such that positive curvature is convex towards the reactants. Hence it is possible to approximate $\int_{V} \dot{w} d V$ as $\int_{V} \rho_{0}\left(S_{L}-\right.$ $\left.D_{M} \kappa_{m}\right)|\nabla c| d V$. The integral $\int_{V} \rho_{0}\left(S_{L}-D_{M} \kappa_{m}\right)|\nabla c| d V$ becomes equal to $\rho_{0} S_{L} A_{T}$ for statistically-planar unity Lewis number flames when $\rho S_{d}=\rho_{0}\left(S_{L}-D_{M} \kappa_{m}\right)$ is used because $\int_{V} \rho_{0} D_{M} \kappa_{m}|\nabla c| d V$ can be written as: $\int_{V} \rho_{0} D_{M} \kappa_{m}|\nabla c| d V \approx \rho_{0} D_{M}\left\langle\kappa_{m}\right\rangle_{s} A_{T}$ (where $\left\langle\kappa_{m}\right\rangle_{s}=$ $\int_{V} \kappa_{m}|\nabla c| d V / \int_{V}|\nabla c| d V$ is the global surface-weighted curvature) and $\rho_{0} D_{M}\left\langle\kappa_{m}\right\rangle_{s} A_{T}$ disappears because of the vanishingly small values of $\left\langle\kappa_{m}\right\rangle_{s}$ resulting from the weak correlation between $|\nabla c|$ and $\kappa_{m}$ [28,29]. However, $\int_{V} \rho_{0} D_{M} \kappa_{m}|\nabla c| d V$ does not vanish for flames with a non-zero mean curvature. Although mean curvature is not huge in comparison to curvature fluctuations, a cylindrical Bunsen flame must have a notable negative mean curvature because of its underlying geometry. The negative mean curvature leads to $\int_{V} \dot{w} d V>\rho_{0} S_{L} A_{T}$, which is reflected in $\langle\Omega\rangle /\langle S\rangle>1$ in Fig. 4. Figure 4 also shows that the expression $\rho_{0} S_{L}^{\text {mod }}=$ 
$\rho_{0}\left(S_{L}-D_{M}\left\langle\kappa_{m}\right\rangle_{s}\right)$, for the Bunsen flames under consideration, represents a good approximation of $\langle\Omega\rangle /\langle S\rangle$. The correlation coefficient between $\langle\Omega\rangle /\langle S\rangle$ and $\rho_{0} S_{L}^{\text {mod }}$ has been found to be close to unity (i.e. 0.98) for the cases considered here and it is worth noting that the correlation coefficient decreases (to 0.84 instead of 0.98 ) if the laminar burning velocity is corrected by stretch instead of curvature. It is worth indicating (not shown) that the results do not change greatly if the mean global curvature $\left\langle\kappa_{m}\right\rangle_{v}=\int_{V} \kappa_{m} d V / V$ is used instead of surface area weighted-global curvature $\left\langle\kappa_{m}\right\rangle_{s}$ for these cases due to the weak correlation between $|\nabla c|$ and $\kappa_{m}$.

A comparison between eqs. $5 \mathrm{i}$ and 5 ii reveals that $I_{0}$ can be exactly expressed as $I_{0}=$ $\overline{\left(\rho S_{d}\right)_{s}} / \rho_{0} S_{L}=S_{L}^{\prime} / S_{L}$ but high-fidelity modelling of $\overline{\left(\rho S_{d}\right)_{S}}$ remains difficult which in turn makes a-priori evaluation of $S_{L}^{\prime}$ difficult. Based on this discussion it can be expected that curvature stretch effects are more pronounced if the mean curvature is not dominated by turbulent curvature fluctuations. It has been shown elsewhere [25] that the width of the curvature PDFs scales approximately with the reciprocal of thermal flame thickness $\delta_{t h}$ which in the context of this work scales as $\delta_{t h} \sim P^{-0.5}$. This suggests that low pressure flames might be more sensitive to mean curvature stretch effects which is consistent with the present observations, as shown in Fig. 3.

It is worth noting that eq. 4 is obtained based on the equilibrium between the reaction rate contribution (i.e. $2[\overline{\dot{w} c}-\overline{\dot{w}} \tilde{c}])$ and the scalar dissipation rate (i.e. $-2 \bar{\rho} \tilde{\varepsilon}_{c}=-2\left[\overline{\rho D_{c} \nabla c . \nabla c}-\right.$ $\left.\bar{\rho} \widetilde{D_{c}} \nabla \tilde{c} . \nabla \tilde{c}\right]$ ) contributions to reaction progress variable variance (i.e. $\widetilde{c^{\prime \prime 2}}=\widetilde{c^{2}}-\tilde{c}^{2}$ ) transport in the flamelet regime of combustion. It has been shown elsewhere [23] that eq. 4 can be derived for $D a \gg 1$ combustion and a scaling analysis was utilised elsewhere $[44,46]$ to demonstrate that this equilibrium is indeed maintained in an order of magnitude sense for 
flamelet combustion with $D a<1$. More information on the scalar dissipation rate modelling can be found in Refs. $[10,23,24,44,46,47]$ and references therein. Thus, eq. 4 does not depend on any assumptions involving the evaluation of flame surface area or the choice of flame speed and as a result $\langle N\rangle$ satisfactorily predicts $\langle\Omega\rangle$ for all cases considered here (see Figs. 3 and 4 ).

\section{CONCLUSIONS}

The applicability of Damköhler's first hypothesis which relates turbulent burning rate (or turbulent flame speed) to turbulent flame area has been assessed based on DNS data for turbulent Bunsen burner flames. It has been found that the proportionality between turbulent burning rate and flame area, expressed respectively as the integrated reaction rate $\int_{V} \dot{w} d V$ and the integrated flame surface density $\int_{V} \Sigma_{\text {gen }} d V=\int_{V}|\nabla c| d V$, does not hold well for turbulent Bunsen flames. By contrast, several previous analyses using statistically planar flames have indicated that $\int_{V} \dot{w} d V$ and $\rho_{0} S_{L} \int_{V}|\nabla c| d V$ are in good agreement. Detailed physical explanations have been provided for the observed discrepancy between $\int_{V} \dot{w} d V$ and $\rho_{0} S_{L} \int_{V}|\nabla c| d V$, and it has been shown that the assumption of $\overline{\left(\rho S_{d}\right)_{s}}=\rho_{0} S_{L}$ yields inaccurate results especially for flames with a non-zero mean curvature. It has been demonstrated also that the evaluation of the two-dimensional reaction progress variable gradient in experimental analyses can considerably amplify the discrepancy between $\int_{V} \dot{w} d V$ and $\rho_{0} S_{L} \int_{V}|\nabla c|_{2 D} d V$. It has been found that $2 \int_{V} \rho N_{c} d V /\left[\left(2 c_{m}-1\right)\right]$ satisfactorily captures the behaviour of $\int_{V} \overline{\dot{w}} d V$ but that the evaluation of $2 \int_{V} \rho D|\nabla c|_{2 D}^{2} d V /\left[\left(2 c_{m}-1\right)\right]$ based on a $2 \mathrm{D}$ projection of the actual reaction progress variable gradient gives rise to an inaccurate estimation of the overall burning rate $\int_{V} \overline{\dot{w}} d V=\rho_{0} S_{T} A_{L}$ and turbulent flame speed $S_{T}$. This analysis offers a derivation of an expression of the Damköhler's first hypothesis from first principles and 
suggests replacement of $S_{L}$ by $S_{L}^{\prime}$, which accounts for volume-integrated, surface areaweighted stretch effects. Furthermore it enables Damköhler's first hypothesis to be valid in general in the flamelet regime, provided turbulent burning rate and flame area are well

represented by the integral values of $\int_{V} \dot{w} d V$ and $\int_{V}|\nabla c| d V$. This, together with the fact that experimental determination of flame area is mostly based on two dimensional slices, suggests that an experimental assessment of Damköhler's first hypothesis remains a challenging task with current state of the art measurement techniques.

\section{ACKNOWLEDGEMENTS}

The authors are grateful to Gauss Centre for Supercomputing / Leibniz Supercomputing Centre (grant: pr74ra) and ARCHER for computing support. 


\section{REFERENCES}

1. K.O. Smith, F.C. Gouldin, Prog. Astro. Aero., 58 (1978) 37-54.

2. R.G. Abdel-Gayed, D. Bradley, M. Lawes, Proc. Roy. Soc. Lond. A., 414 (1987) 389-413.

3. O.L. Gülder, Proc. Combust. Inst., 23 (1990) 743-750.

4. K.N.C. Bray, Studies of turbulent burning velocity, Proc. R.Soc. Lond, A 431 (1990) 315335.

5. V.L. Zimont, Exp. Therm. Fluid Sci., 21 (2000) 179-186.

6. N. Peters, J. Fluid Mech., 384 (1999) 107-132.

7. A.N. Lipatnikov, J. Chomiak, Prog. Energy Combust. Sci., 28 (2002) 1-74.

8. S.P.R. Muppala, N.K. Aluri, F. Dinkelacker, A. Leipertz, Combust. Flame, 140 (2005) 257266.

9. V.R. Savarianandam, C.J. Lawn, Combust. Flame, 146 (2006) 1-18.

10. H. Kolla, J.W. Rogerson, N. Chakraborty, N. Swaminathan, Combust. Sci. Technol., 181 (2009) 518-535.

11. G. Damköhler, Zeitschrift für Elektrochemie und angewandte physikalische Chemie, 46 (1940) 601-626.

12. J. Daou, J. Dold, M. Matalon, Combust. Theor. Modell, 6 (2002) 141-153.

13. J. Daou, P. Sparks, Combust. Theor. Modell, 11 (2007) 697-704.

14. R.S. Cant, G. Nivarti, Phys. Fluids, 29 (2017) 085107.

15. M. Boger, D. Veynante, H. Boughanem, A. Trouvé, Proc. Combust. Inst., 27 (1998) 917925.

16. O. Gülder, Proc. Combust. Inst., 31 (2007) 1369-1375.

17. T.C. Chew R.E. Britter, K.N.C. Bray, Combust. Flame, 75 (1989) 165-174.

18. H. Kobayashi, T. Nakashima, T. Tamura, K. Maruta, T. Niioka, Combust. Flame, 108, (1997) 104-110. 
19. O.L. Gülder, G.J. Smallwood, R. Wong, et al., Combust. Flame, 120 (2000) 407-416.

20. Y.-C. Chen, R.W. Bilger, Combust. Flame, 131(2002) 400-435.

21. M. Zhang, J. Wanga, W. Jin, Z. Huang, H. Kobayashi, L. Mac, Combust. Flame, 162 (2015) 2087-2097.

22. R.S. Cant, K.N.C. Bray, Proc. Combust. Inst., 22 (1988) 791-799.

23. K.N.C. Bray, Turbulent flows with premixed reactants, in Turbulent Reacting Flows, Springer Verlag, Berlin Heidelburg, New York, eds. P.A. Libby, F.A. Williams, (1980) 115183.

24. N. Chakraborty, R.S. Cant, Combust. Flame, 158 (2011) 1768-1787.

25. M. Klein, H. Nachtigal, M. Hansinger, M. Pfitzner, N. Chakraborty, Proc. 10th Mediterranean Symposium (2017).

26. T. Echekki, J.H. Chen, Combust. Flame, 106 (1996) 184-202.

27. N. Peters, P. Terhoeven, J.H. Chen, T. Echekki, Proc. Combust. Inst., 27 (1998) 833-839.

28. N. Chakraborty, S. Cant, Combust. Flame, 137 (2004) 129-147.

29. N. Chakraborty, M. Klein, R.S. Cant, Proc. Combust. Inst., 31 (2007) 1385-1392.

30. J. Lai, M. Klein and N. Chakraborty, Flow Turb. Combust., (2018), https://doi.org/10.1007/s10494-018-9907-5.

31. M. Klein, C. Kasten, N. Chakraborty, N. Mukhadiyev and H.G. Im. Combust. Theor. Modell, (2018) accepted.

32. S.R. Turns, An introduction to combustion: concepts and applications, 3rd Edition, McGraw Hill (2011).

33. N. Peters, Turbulent Combustion, Cambridge Monograph on Mechanics, Cambridge University Press, Cambridge (2000). 
34. K.W. Jenkins, R.S. Cant, DNS of turbulent flame kernels, In C. Liu, L. Sakell and T. Beautner (Eds.), Proc. $2^{\text {nd }}$ AFOSR Conf. on DNS and LES, Kluwer Academic Publishers, (1999) 192-202.

35. M. Klein, A. Sadiki, J. Janicka, J. Comp. Phys., 186 (2003) 652-665.

36. T. Poinsot, S.K. Lele, J. Comp. Phys. 101 (1992) 104-129.

37. S.B. Pope, Int. J. Engg. Sci., 26 (1988) 445-469

38. E. R. Hawkes, R. Sankaran, and J. H. Chen, Proc. Combust. Inst., 33 (2011) 1447-1454.

39. N. Chakraborty, E.R. Hawkes, Phys. Fluids, 23 (2011) 065113.

40. N. Chakraborty, R.S. Cant, Proc. Combust. Inst., 32 (2009) 1445-1453.

41. V. Sabelnikov, A.N. Lipatnikov, N. Chakraborty, S. Nishiki, T. Hasagawa, Proc. Combust. Inst., 36 (2017) 1893-1901.

42. R. Farran, N. Chakraborty, Entropy, 15 (2013) 1540-1566.

43. G. Nivarti, S. Cant, Proc. Combust. Inst., 36 (2017) 1903-1910.

44. N. Chakraborty, R.S.Cant, Combust. Flame, 158 (2011) 1768-1787.

45. S. Chaudhuri, F. Wu and C.K. Law, Physical Review E, 88 (2013) 033005.

46. N. Chakraborty, N. Swaminathan, Flow Turb. Combust., 87 (2011) 261-292.

47. N. Chakraborty, M. Champion, A. Mura, N. Swaminathan, Scalar dissipation rate approach to reaction rate closure, Turbulent premixed flame, (Eds. N. Swaminathan, K.N.C. Bray), Cambridge University Press, 1st Edition, Cambridge, UK (2011) 74-102. 


\section{FIGURE CAPTIONS}

Fig. 1: The inlet conditions of Bunsen burner flames on the combustion regime diagram.

Fig. 2: Instantaneous view of (a) reaction progress variable $c$ (1st column) and (b) normalised velocity magnitude $|\vec{u}| / S_{L}$ (2nd column) in the central mid-plane for cases $\mathrm{A}, \mathrm{C}$ and $\mathrm{E}$.

Fig. 3: Variations of $\langle\Omega\rangle,\langle S\rangle,\left\langle S_{2 D}\right\rangle,\langle N\rangle,\left\langle N_{2 D}\right\rangle$ for cases A-I based on averaging different realisations.

Fig. 4: Variations of $\langle\Omega\rangle /\langle S\rangle, \pi\langle\Omega\rangle / 4\left\langle S_{2 D}\right\rangle,\langle\Omega\rangle /\langle N\rangle$ and $\rho_{0} S_{L}^{\text {mod }}$ for cases A-I.

Fig. 5: $\rho S_{d}|\nabla c| \times \delta_{t h} / \rho_{0} S_{L}$ and $\rho_{0} S_{L}|\nabla c| \times \delta_{t h} / \rho_{0} S_{L}$ conditional upon $c$ for cases A and C. The bars indicate the standard deviation of the respective quantities. 